\title{
Size and thermal effects on sedimentation behaviors of two spheres
}

Bo Yang ${ }^{1}$, Sheng Chen ${ }^{* 1,2,3}$, Yan Xiong ${ }^{1}$, Rui Zhang ${ }^{1}$, Chuguang Zheng ${ }^{1}$

1 State Key Laboratory of Coal Combustion, School of Energy and Power Engineering, Huazhong University of Science and Technology, Wuhan, 430074, China

2 Institute for Modelling and Simulation in Fluodynamics, Nanoscience and Industrial Mathematics "Gregorio Millan Barbany", Universidad Carlos III de Madrid, Leganes 28911, Spain

3 Faculty of Engineering, The University of Nottingham, University Park, Nottingham NG7 2RD, UK

*Corresponding author: Faculty of Engineering, University of Nottingham.

E-mail address: shengchen.hust@gmail.com

\begin{abstract}
Gas-solid flows are commonly found in nature, as well as in industries. In such flows the size of the solid particles generally is not uniform. In addition, usually there is heat transfer between solid particles and gas flows. The hydrodynamics and heat transfer both make the behavior of gas-solid flows extremely complicated. In order to reveal these effects, in this paper three cases: (1) two isothermal, (2) two hot and (3) two cold spherical particles with various size ratios are investigated using lattice Boltzmann method-immersed boundary (LB-IB). It is observed that, for the first time, the tumbling duration of both two hot particles and two cold particles settling in vertical channel, is prolonged with size ratio increasing. The differences of threshold size ratio among the three cases are significant and the threshold size ratio of two hot particles is the largest one. Especially, it is found that heat transfer affects critically the interaction of two hot particles with low size ratios. In addition, against particle size ratio increasing, heat transfer effects on the interaction between two non-identical particles become weak.
\end{abstract}




\section{Introduction}

Sedimentation of solid particles in a viscous fluid exists in many natural and biological situations as well as in industrial applications, such as sand deposition in rivers, drug delivery in blood flows, particle fluidization in fluidized bed reactors. Over the past decades, the sedimentation of particles with/without heat transfer has been studied extensively using various numerical and experimental methods. Fortes et al. [1] firstly investigated the sedimenting behavior of isothermal spherical particles. An important phenomenon called as "drafting, kissing and tumbling" (DKT) was found for the first time. Feng et al. [2] investigated particle-fluid, particle-wall and particle-particle interaction during the sedimentation of two isothermal particles. They demonstrated that interaction mechanism for particulate flow was associated with lubrication, long bodies, and wakes. Gan et al. [3] investigated the effects of heat transfer on the behaviors of double circular particles during sedimentation. They found that cold particles would tend to repel each other while hot particles attract. Zahra Hashemi et al. [4] investigated sedimenting behavior of 30 hot particles under gravity in a newtonian fluid. Hydraulic and thermal convection interactions between particles and surrounding fluid were revealed. Effects of particle deformation and particle-fluid heat transfer on particle-particle interactions were studied by Henrik Ström et al. [5]. Cao et al. [6] comprehensively studied the effects of initial particle position arrangement on sedimentation of two isothermal particles. They identified three interaction regimes (repulsion, attraction and transition regime) between two settling particles based on initial configurations. They also investigated the effects of heat transfer on sedimentation of two cold particles in each above regime [7].

However, attentions given to the effects of size difference between sedimenting particles on the particulate flows were limited. Johnson et al. [7] investigated the behaviors of 101 spheres with random sizes falling in a liquid-filled tube. The simulation for interaction dynamics of two spheres in a finite fluid-filled rotating cylinder was reported in Mukundakrishnan et al. [9]. They compared the behaviors of two non-identical particles with those of identical particles. For two non-identical 
particles, the smaller particle could execute a spiralling motion while the larger was in near-circular orbital motion. Shao et al. [10] simulated the interactions between two circular particles with different sizes sedimenting in a two-dimensional channel. Their results demonstrated that only for small diameter ratio, the two particles would undergo repeated DKT process. The interaction effects on motion of the small particle were stronger than that of the large particle. Wang et al. [11] studied the size effects on the DKT phenomenon of two non-identical particles sedimenting in a two-dimensional infinite channel. The influences of initial position distribution and diameter ratio on the behaviors of the two particles were investigated. They found that two particles with different sizes were easier to separate than two identical ones. Recently, Liao et al. [12] numerically investigated the hydrodynamic interactions of two spheres with different sizes and initial configurations using lattice Boltzmann method-immersed boundary method (LB-IB). When a regular sphere was placed below the larger one, the duration of kissing decreased against increasing diameter ratio. While the regular sphere was placed above the larger one, the duration of the kissing increased with increasing diameter ratio. They also investigated the threshold diameter ratio of two isothermal particles when the regular sphere was placed above the larger one. They found that there was no DKT interaction beyond the threshold diameter ratio. However, all studies mentioned above were limited to the sedimentation of non-identical particles without considering heat transfer between particle and fluid.

In order to reveal the combination effects of heat transfer and non-identical size of particles, in this paper, size ratio effects on the dynamic behaviors of two non-isothermal spheres are simulated for the first time. In order to provide a clear comparison, their isothermal counterpart is investigated, too. The threshold diameter ratio of two non-isothermal particles is also studied firstly. The rest of this paper is organized as follows. Section 2 gives a brief introduction for numerical method used here. The code is validated in Section 3. Further, the size effects on the hydrodynamic interactions of two non-isothermal spheres are investigated in Section 4. Finally, some concluding remarks are listed in Section 5. 


\section{Numerical method}

Over the years, the lattice Boltzmann method (LBM) has been developed to an alternative and efficient tool for simulating particulate flow due to its capability of treating moving boundary [13]-[16]. As a discrete form of Boltzmann equation, lattice Boltzmann equation can recover to Navier-Stokes (N-S) equations using multi-scaling analysis. Here we will employ D3Q19 LB model to solve both flow and temperature field.

The evolution equations for flow and temperature field with external terms are formulated as

$$
\begin{aligned}
& f_{i}\left(\mathbf{x}+\mathbf{c}_{i} \Delta t, t+\Delta t\right)-f_{i}(\mathbf{x}, t)=-\frac{1}{\tau_{f}}\left[f_{i}(\mathbf{x}, t)-f_{i}^{e q}(\mathbf{x}, t)\right]+F_{i} \Delta t \\
& T_{i}\left(\mathbf{x}+\mathbf{c}_{i} \Delta t, t+\Delta t\right)-T_{i}(\mathbf{x}, t)=-\frac{1}{\tau_{T}}\left[T_{i}(\mathbf{x}, t)-T_{i}^{e q}(\mathbf{x}, t)\right]+Q_{i} \Delta t
\end{aligned}
$$

where $f_{\mathrm{i}}, T_{\mathrm{i}}$ are distribution functions for flow and temperature field respectively, and $F_{i}, Q_{i}$ are discrete terms for flow and temperature evolution equations respectively. $\Delta \mathrm{t}=\mathrm{dx} / \mathrm{c}$ is the time interval where $\mathrm{dx}$ is the lattice spacing and $\mathrm{c}$ is the lattice speed. The equilibrium distribution functions $f^{e q}$ and $T^{e q}$ are defined as

$$
\begin{aligned}
& f_{i}^{e q}=\omega_{i} \rho\left[1+3 \frac{\mathbf{c}_{i} \cdot u}{c^{2}}+4.5 \frac{\left(\mathbf{c}_{i} \cdot u\right)^{2}}{c^{4}}-1.5 \frac{u^{2}}{c^{2}}\right] \\
& T_{i}^{e q}=\omega_{i} T\left[1+3 \frac{\mathbf{c}_{i} \cdot u}{c^{2}}+4.5 \frac{\left(\mathbf{c}_{i} \cdot u\right)^{2}}{c^{4}}-1.5 \frac{u^{2}}{c^{2}}\right]
\end{aligned}
$$

$\omega_{i}$ is weighting coefficient and for D3Q19 model is defined as

$$
\left\{\begin{array}{cc}
\omega_{i}=1 / 3 & \mathrm{i}=0 \\
\omega_{i}=1 / 18 & \mathrm{i}=1 \sim 6 \\
\omega_{i}=1 / 36 & \mathrm{i}=7 \sim 18
\end{array}\right.
$$

Using forcing scheme proposed by Guo et al. [17], $F_{i}$ and $Q_{i}$ are defined by

$$
\begin{aligned}
& F_{i}=\omega_{i}\left(1-\frac{1}{2 \tau_{f}}\right)\left[\frac{\mathbf{c}_{i}-\mathbf{u}}{c_{s}^{2}}+\frac{\mathbf{c}_{i} \cdot \mathbf{u}}{c_{s}^{4}} \mathbf{c}_{i}\right] \cdot \mathbf{F} \\
& Q_{i}=\omega_{i}\left(1-\frac{1}{2 \tau_{T}}\right) Q
\end{aligned}
$$


where $\mathbf{F}=\mathbf{F}_{b}+\mathbf{F}_{T}$ is the total force acting on the fluid node, $\tau_{\mathrm{f}}, \tau_{\mathrm{T}}$ are relaxation times for flow and temperature field respectively. $\mathbf{F}_{T}$ can be obtained using Boussinesq approximation

$$
\mathbf{F}_{T}=-\rho_{0} \beta\left(T-T_{0}\right) \mathbf{g}
$$

where $\rho_{0}, T_{0}$ are reference density and reference temperature respectively, $\mathbf{g}$ is the gravity constant, and $\beta$ is the thermal expansion coefficient, which is defined by

$$
\beta=\frac{G r \cdot v^{2}}{D^{3} g \Delta T}
$$

where $\Delta \mathrm{T}=\mathrm{T}_{\mathrm{s}}-\mathrm{T}_{\mathrm{f} 0}$ is temperature difference between solid particle and fluid, $D$ is the diameter of solid particle, and $v$ is the kinematic viscosity coefficient. The Prandtl number $\operatorname{Pr}=v / \alpha$ is related to relaxation times $\tau_{\mathrm{f}}, \tau_{\mathrm{T}}$ by $\tau_{\mathrm{f}}=3 \mathrm{v} /\left(\mathrm{dx} *_{\mathrm{c}}\right)+0.5$ and $\tau_{\mathrm{T}}=3 \alpha /(\mathrm{dx} * \mathrm{c})+0.5$, where $\alpha$ is the thermal diffusion coefficient.

The macroscopic variables can be calculated as

$$
\rho=\sum_{i=0} f_{i}, \rho \mathbf{u}=\sum_{i=0} \mathbf{c}_{i} f_{i}+\frac{\Delta t}{2} \mathbf{F}, T=\sum_{i=0} T_{i}+\frac{\Delta t}{2} Q
$$

Macroscopic governing equations can be recovered from the lattice Boltzmann equations using Chapman-Enskog analysis as following:

$$
\begin{aligned}
& \frac{\partial \rho}{\partial t}+\nabla \cdot \rho \mathbf{u}=0 \\
& \frac{\partial}{\partial t}(\rho \mathbf{u})+\nabla \cdot(\rho \mathbf{u u})=-\nabla p+v \nabla \cdot\left[\rho \nabla \mathbf{u}+(\nabla \mathbf{u})^{T}\right]+\mathbf{F} \\
& \frac{\partial T}{\partial t}+\nabla \cdot(\mathrm{T} \mathbf{u})=\alpha \nabla^{2} T+Q
\end{aligned}
$$

A direct-forcing method with sharp interface scheme proposed by Kang et al. [18]-[19] is applied to solve particle boundary for both flow and temperature field because of its sufficient precision and simply implementing. We firstly combine the original two-dimensional direct-forcing method proposed by Kang et al. [18]-[19] with the three-dimensional interpolation scheme proposed by Kim et al. [20]. For the interpolation scheme, forcing nodes are located inside the solid and closest to the boundary, as shown in Fig. 1. Here nodes O-G are lattice nodes used in simulation and forcing nodes are marked by subscript $\mathrm{O}$. $\mathbf{U}_{\mathrm{o}}$ indicates the no-slip velocity at solid node $\mathrm{O}$, and can be obtained by interpolating through adjacent fluid nodes. Based on 
distributions of fluid and solid nodes around particle boundary, three situations are considered. (a) Boundary node $\mathrm{P}_{1}\left(\mathrm{P}_{1}\right.$ locates inside cube OABCDEFG) is on the boundary where straight line $\mathrm{OP}_{1}$ perpendicularly intersects with the boundary. Trilinear interpolation is applied as

$$
\begin{array}{r}
\mathbf{U}=\frac{1}{(1-\Delta y)(1-\Delta x)(1-\Delta z)}\left[\mathbf{U}_{b}-\Delta y(1-\Delta x)(1-\Delta z) \mathbf{u}_{A}-\Delta x(1-\Delta y)(1-\Delta z) \mathbf{u}_{G}-\Delta z(1-\Delta y)(1-\Delta x) \mathbf{u}_{C}\right. \\
\left.-\Delta x \Delta y(1-\Delta z) \mathbf{u}_{F}-\Delta z \Delta y(1-\Delta x) \mathbf{u}_{B}-\Delta x \Delta z(1-\Delta y) \mathbf{u}_{D}-\Delta z \Delta y \Delta x \mathbf{u}_{E}\right]
\end{array}
$$

where $\mathbf{U}_{\mathrm{b}}$ is the particle velocity at node $\mathrm{P}_{1}, \Delta \mathrm{x}, \Delta \mathrm{y}, \Delta \mathrm{z}$ are distance components between nodes $\mathrm{P}_{1}$ and $\mathrm{O}$ in $\mathrm{x}-, \mathrm{y}$ - and $\mathrm{z}$-direction. $\mathbf{u}_{\mathrm{A}-\mathrm{G}}$ indicate the fluid velocity at nodes A-G. (b) $\mathrm{P}_{2}$ locates on plane $\mathrm{OABC}$, and bilinear interpolation is applied as

$$
\mathbf{U}_{O}=\frac{1}{\Delta y \Delta z}\left\{\mathbf{U}_{b}-\left[\Delta z(1-\Delta y) \mathbf{u}_{C}+\Delta y \Delta z \mathbf{u}_{B}+(1-\Delta z) \Delta y \mathbf{u}_{A}\right\}\right.
$$

Note that the boundary node $\mathrm{P}_{2}$ may locate on other adjacent planes OAFG, OCDG and thus three situations must be considered in applications. (c) $\mathrm{P}_{3}$ locates on line segment $\mathrm{OC}$ (or OA, OG) and linear interpolation is applied as

$$
\mathbf{U}_{O}=\left\{\begin{array}{cc}
\frac{1}{\Delta} \mathbf{U}_{\mathrm{b}}-\frac{1-\Delta}{\Delta} \mathbf{u}_{C} & \text { if } \Delta \geq 0.5 \\
2 \mathbf{U}_{\mathrm{b}}-2 \Delta \mathbf{u}_{C}-(1-2 \Delta) \mathbf{u}_{C^{\prime}} & \text { if } \Delta<0.5
\end{array}\right.
$$

where $\Delta$ indicates distance $\mathrm{CP}_{3}$ and $C^{\prime}$ indicates the node obtained by advancing node $\mathrm{C}$ a lattice in $\mathrm{OC}$ direction.

The force density $\mathbf{F}_{\mathrm{b}}$ acting on forcing node $\mathbf{O}$ is calculated by

$$
\mathbf{F}_{b}=2 \rho \frac{\mathbf{U}_{O}-\mathbf{u}_{O}}{\Delta t}
$$

where $\mathbf{u}_{\mathrm{o}}, \rho$ indicate the desired fluid velocity and density at forcing node $\mathbf{O}$ respectively (namely $\rho=\sum f_{i}, \mathbf{u}=1 / \rho \sum f_{i} \boldsymbol{c}_{i}$ ).

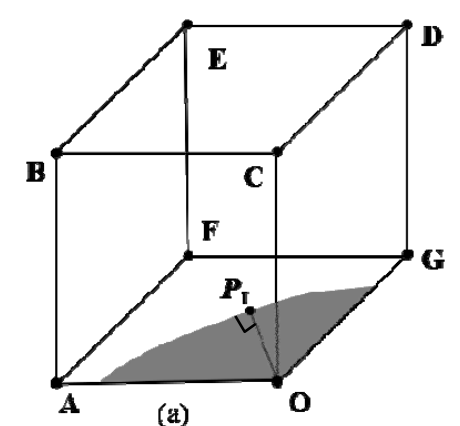

(a)

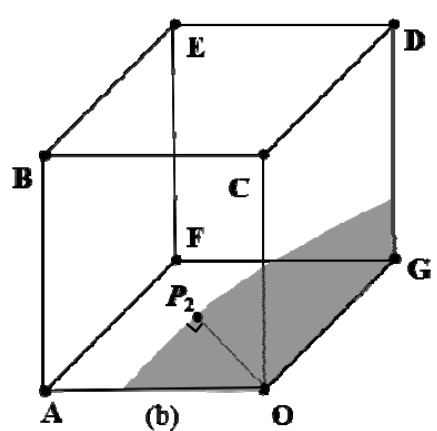

(b)



(c) 
Fig. 1 Schematic diagram for the interpolation scheme in three dimension: (a) trilinear interpolation ( $\mathrm{P}_{1}$ locates inside the cube); (b) bilinear interpolation $\left(\mathrm{P}_{2}\right.$ locates on plane $\left.\mathrm{OABC}\right)$; (c) linear interpolation $\left(\mathrm{P}_{3}\right.$ locates on line segment $\left.\mathrm{OC}\right)$. Here, shaded sections represent the region covered by solid particle. Lines $\mathrm{OA}, \mathrm{OC}$, OG represent $\mathrm{X}, \mathrm{Y}, \mathrm{Z}$ coordinate direction respectively.

Note that the same strategy is used to obtain the source in solid points for energy equation, such as energy forcing term $\mathrm{Q}$.

Newtonian dynamic equations are solved to obtain the motion of particles as following:

$$
\begin{aligned}
& M_{i} \frac{d U_{i}(t)}{d t}=F_{i}(t) \\
& I_{i} \cdot \frac{\Omega_{i}(t)}{d t}+\Omega_{i}(t) \times\left[I_{i} \cdot \Omega_{i}(t)\right]=T p_{i}(t)
\end{aligned}
$$

where $M_{i}, I_{i}$ are mass and inertial tensor of the $i$ th particle, $\mathbf{U}_{i}, \boldsymbol{\Omega}_{i}$ are velocity and angle velocity of the $i$ th particle, and $\mathbf{F}_{i}, T p_{i}$ are total force and torque implemented on the $i$ th particle. When two solid particles come into close contact with each other, a lubrication force is introduced. As pointed out by Kromkamp et al. [21], this force is caused by the attenuation of the fluid film in the gap between the two particles and is repulsive upon approach and attractive upon separation of the particles. The lubrication force for 3D systems is calculated as [22]

$$
\begin{aligned}
& \mathbf{F}=-6 \pi \eta \mathbf{U}_{12} \cdot \hat{\mathbf{R}}_{12} \frac{r_{1} r_{2}}{\left(r_{1}+r_{2}\right)^{2}}\left(\frac{1}{h}-\frac{1}{h_{c}}\right), \mathrm{h}<\mathrm{h}_{\mathrm{c}} \\
& \mathbf{F}=\mathbf{0}, \quad \mathrm{h}<0
\end{aligned}
$$

where $\eta$ is the average viscosity of fluid, $\mathbf{U}_{12}$ is the velocity of particle 2 relative to particle $1, \hat{\mathbf{R}}_{12}$ is the unit vector from the center of particle 1 toward particle 2 . For a 3D system, the cut-off distance $h_{c}$ between the particle surfaces was chosen to be 1.1 lattice units. $r_{1}, r_{2}$ are radiuses of the two particles. According to Ref. [22], this model leads to more accurate results for particle interactions at short inter-particle distances without causing instabilities in the particle dynamics. Note that the lubrication force model avoids the physical contact between two particles by implementing a strong repulse force on the two particles. 
In practical applications, following steps are executed:

1) Initialize the velocity and temperature field, and the positions of solid particles.

2) Calculate the fluid velocity $\mathbf{u}$ and density $\rho$ by Eq. 10 without forcing term.

3) Calculate the boundary force density $\boldsymbol{F}_{\mathrm{b}}$ by Equations 14-17, and update $\mathbf{u}$ and $\rho$ by Eq. 10 .

4) Execute the evolution step using Eq. 1.

5) Execute the same steps 2-4 for temperature field.

6) Calculate the total force and torque on particles and update the positions and velocities of particles.

\section{Numerical validation}

The reliability of our numerical code to treat particle-particle interaction has been validated in our previous work [6]. So in this section, we only validate the heat transfer part of our code.

The case studied by Dan and Wachs [23] and Hashemi et al. [4] is used here as a benchmark test to validate the capability of our code for simulating thermal sphere particles settling in channel. Three cases are considered: one cold particle $(\mathrm{Gr}=-100)$, one isothermal particle $(\mathrm{Gr}=0)$ and one hot particle $(\mathrm{Gr}=100)$. Most simulation parameters are set the same as those in Ref. [23] and Ref. [4]. Except that the fluid temperature is set to 0 and particle temperatures are set to $-1,0$ and 1 respectively in the above three cases, which is based on the dimensionless criteria. Fig. 2 describes the variations of dimensionless particle terminal velocity with $R e$ at various $G r$. Here the terminal velocity is normalized by the reference velocity $U_{r e f}=\sqrt{8 R\left(\rho_{r}-1\right) g / 3}$, where $R$ is the radius of settling particle, $\rho_{\mathrm{r}}$ is the density ratio between particle and fluid and $g$ is gravity constant. As $\operatorname{Re}<100$, the terminal velocity of cold particle is larger than that of isothermal particle and that of hot particle. While as $\mathrm{Re} \geq 100$, for the three cases the terminal velocities are nearly consistent. These phenomena stem from the competition between buoyant force and inertia force. Fig. 2 indicates good agreement between our results and results from Dan \& Wachs [23]. 


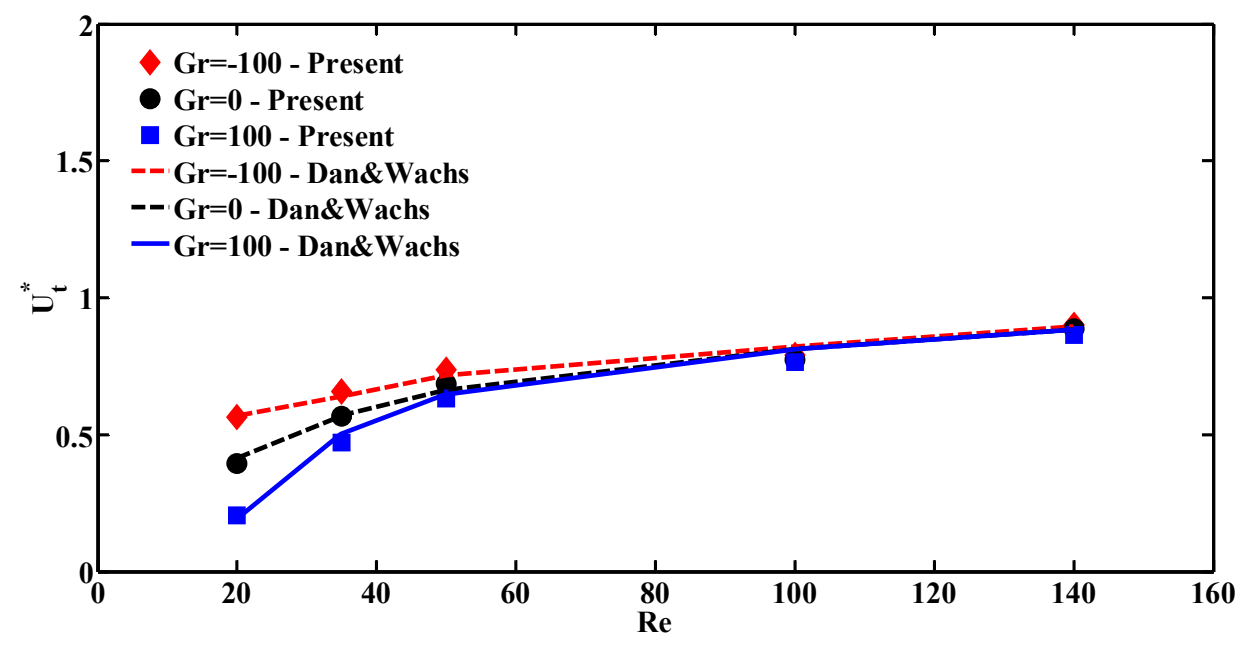

Fig. 2 Variations of dimensionless particle terminal velocity with $\mathrm{Re}$ at $\mathrm{Gr}=-100$,

0, 100: comparison between present and results of Dan \& Wachs [23].

\section{Results and discussion}

In this section, size effects on the sedimentation of two isothermal and nonisothermal spherical particles in a semi-infinite channel are investigated. The particle diameter size and channel size are $D_{0}=1.0 \mathrm{~cm}$ and $L x=4.0 D_{0}, L y=4.0 D_{0}, L z=10.0 D_{0}$ respectively. Lattice spacing $d x=D_{0} / 32$. Initially the two particles are assigned to vertical arrangement with a center distance of $2 D_{0}$, as shown in Fig. 3. The particles and fluid have a density of $1.1 \mathrm{~g} / \mathrm{cm}^{3}$ and $1.0 \mathrm{~g} / \mathrm{cm}^{3}$ respectively. The kinematic viscosity of fluid $v$ is $0.1 \mathrm{~cm}^{2} / \mathrm{s}$. The lattice speed $\mathrm{c}=100 \mathrm{~cm} / \mathrm{s}$ and gravity constant $\mathrm{g}=-981 \mathrm{~cm} / \mathrm{s}^{2}$. The Grashof numbers $G r$ is fixed to 1000 and Prandtl number $P r$ is fixed to 1.0. We fix one particle diameter to $D_{0}$ and adjust diameter ratio $\alpha$ to obtain another particle diameter $D_{a}$ by $D_{a}=\alpha^{*} D_{0}$. Here the adjustable-size particle is placed above the regular one unless otherwise specified. The two particles are initially located on the plane $\mathrm{Y}=2.0 D_{0}$, which is referred as principal plane here and hereafter. At lattice time $\mathrm{t}=0$, the two particles start to accelerate under gravity. During the simulation, we apply a shifting method [24] to solve infinite length in settling direction. When particles settle down 10 lattices in settling direction, the top 10 layers of lattice are abandoned and 10 layers of lattice initialized by initial condition are 
added to channel bottom.

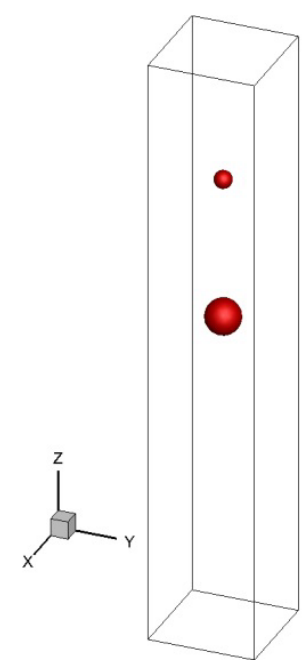

Fig. 3 Simulation schematic

\subsection{Sedimentation of two isothermal particles with different sizes}

In this subsection, the size effects on the sedimentation of two isothermal particles are investigated. We find that larger size ratio produces longer duration of tumbling process. We take two ratios $(\alpha=0.7$ and $\alpha=1.0)$ as the examples to illustrate it. Variations of dimensionless spacing $(L / D)$ of two isothermal particles at $\alpha=0.7$ and at $\alpha=1.0$ are shown in Fig. 4(a). With increasing size ratio, kissing occurs earlier and tumbling duration is prolonged. The observation is consistent with that reported in Ref. [12]. When $\alpha=0.7$, the smaller particle settles slowly than the larger one and thus distance between the two particles is increased before drafting. Fig. 4(b) depicts variations of vertical velocities of two isothermal particles at $\alpha=0.7$ and $\alpha=1.0$. Here and hereafter, P1, P2 indicate the trailing (upper) particle and the leading (lower) one respectively. In our simulations, the two particles move only on the $\mathrm{X}-\mathrm{Z}$ plane due to symmetry. When $\alpha=0.7, \mathbf{P 1}$ settles slowly and spends more time to catch up with $\mathbf{P 2}$ than their $\alpha=1.0$ counterpart. At the kissing instant $\mathbf{P 1}$ has a larger settling velocity at $\alpha=0.7$ than at $\alpha=1.0$. For $\alpha=1.0$, two isothermal particles experience much stronger lubrication force during tumbling stage than their $\alpha=0.7$ counterparts. When $\alpha=1.0$, in separating stage, $\mathbf{P 2}$ undergoes strong repulsion force and obtains a small velocity. Subsequently $\mathbf{P 2}$ accelerates and P1 decelerates, which leads to the distance decreasing process shown in Fig. 4(a). These phenomena are very different from their 
$\alpha=0.7$ counterparts. Fig. 4(c) depicts the variations of horizontal velocities of two isothermal particles at $\alpha=0.7$ and at $\alpha=1.0$, respectively. In separating stage two particles firstly accelerate to two side walls, respectively, and then decelerate due to wall effects [2]. For $\alpha=1.0$, the acceleration time of $\mathbf{P 2}$ is prolonged and the wall effects on $\mathbf{P} 2$ are much stronger than their $\alpha=0.7$ counterparts.
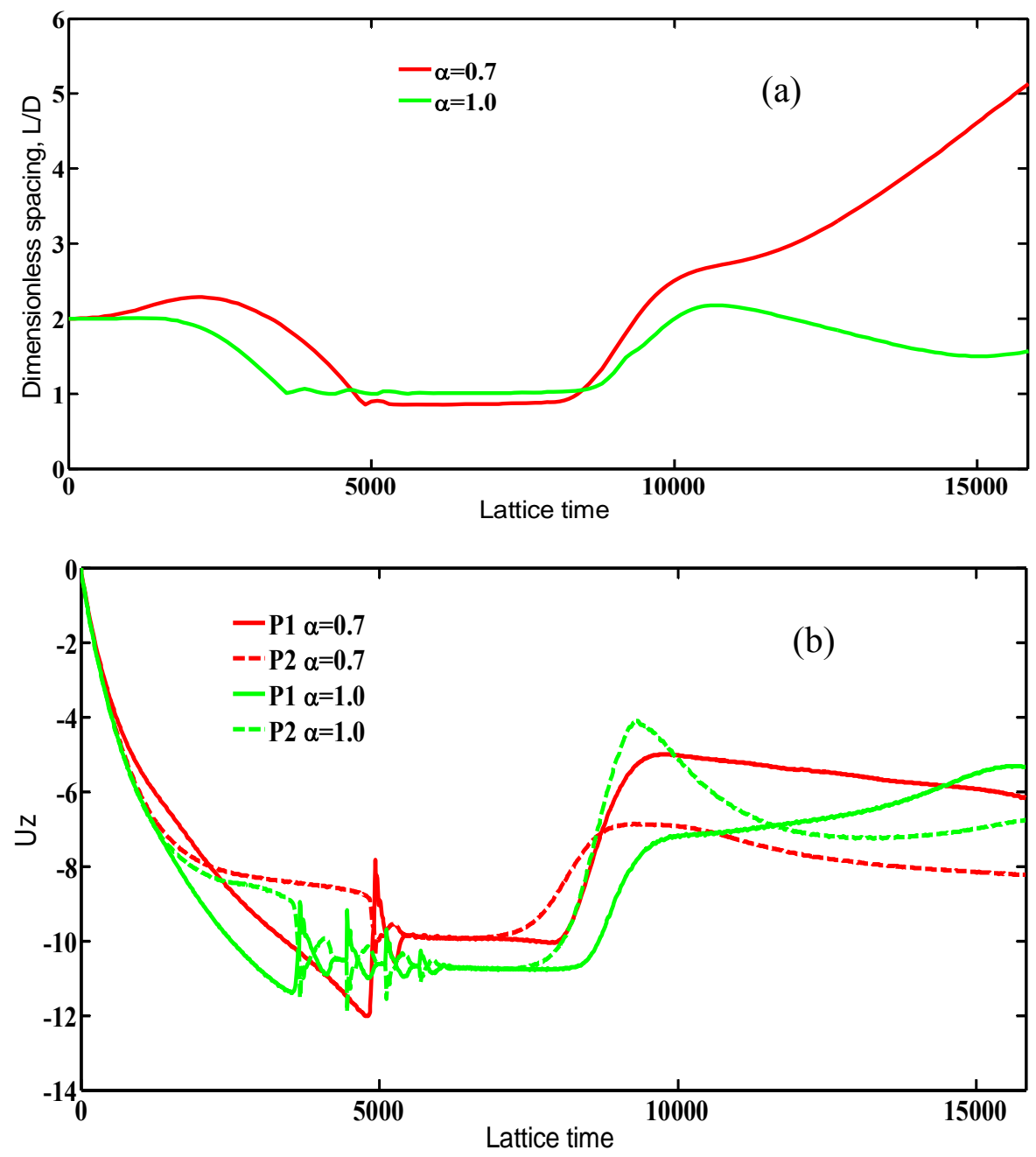


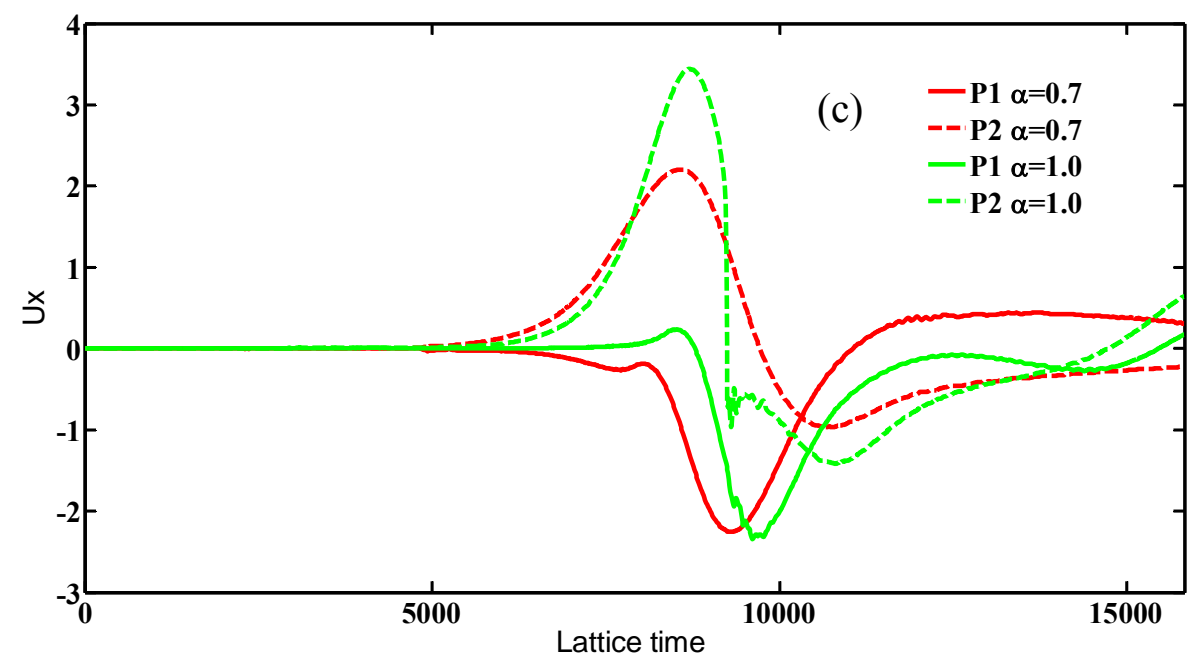

Fig. 4 Variations of (a) dimensionless distance (b) Z- and (c) X-direction velocities of two isothermal particles at $\alpha=0.7$ and at $\alpha=1.0$.

\subsection{Sedimentation of two cold particles with different sizes}

In this subsection, size effects on the sedimentation of two cold particles are investigated. We take the same ratios $(\alpha=0.7$ and $\alpha=1.0)$ as the examples for this investigation. Fig. 5(a) depicts the DKT processes between two cold particles at $\alpha=0.7$ and $\alpha=1.0$. One can see that the duration of tumbling increases with particle size ratio $\alpha$. This result is consistent with their isothermal particles discussed above. Moreover their kissing process occurs earlier and separating instant is postponed. When $\alpha=0.7$, after separating stage distance between the two cold particles increases sharply. Fig. 5(b) shows the variations of Z-direction velocities of two cold particles at $\alpha=0.7$ and at $\alpha=1.0$, respectively. For $\alpha=1.0$, during tumbling process settling velocities of the two cold particles fluctuate more vigorously than their $\alpha=0.7$ counterparts. It indicates that for $\alpha=1.0$ the two cold particles experience stronger lubrication force than their $\alpha=0.7$ counterparts. After separating stage $\mathbf{P} 2$ accelerates and thus settles fast than $\mathbf{P 1}$. Consequently distance between the two cold particles at $\alpha=1.0$ is reduced, as shown in Fig. 5(a). Fig. 5(c) shows variations of the X-direction velocity of two cold particles at $\alpha=0.7$ and at $\alpha=1.0$. For $\alpha=1.0$, during the early stage of tumbling X-direction velocities of two cold particles nearly maintain zeroes. Such phenomenon indicates their relative vertical position will not alter during the early period of tumbling. 
Fig. 6 describes distributions of Z-direction velocity, temperature and pressure at lattice time $\mathrm{t}=7000$ at $\alpha=0.7$. As shown in

Fig. 6, the small particle (P1) is completely sucked into a low pressure area with cold, downward stream, which will induce particle acceleration. Consequently the small particle (P1) settles fast than the larger one (P2) and subsequently separating process happens, as shown in Fig. 5(a) and (b). The variations of drag coefficient, $C_{d}=F_{w} /\left(0.5^{*} U_{w} * U_{w} * D\right)$, are depicted in Fig. 7, where $F_{w}, U_{w}$ are resistances on particles and particle velocities in settling direction (Z-direction), respectively. For $\alpha=0.7$, before separating stage drag coefficient of $\mathbf{P 1}$ is lower than $\mathbf{P 2}$. In addition, when lubrication force becomes dominant, fluctuations happen. After separating stage their drag coefficient curves are nearly identical and the fluctuations are very weak. The two curves at $\alpha=1.0$ are somewhat similar to their corresponding settling velocity curves shown in Fig. 5(b). Variations of Nusselt numbers $N u$ of two cold particles with time at $\alpha=0.7$ and at $\alpha=1.0$ are plotted in Fig. 8. For $\alpha=0.7$, during initial sedimentation P1 settles slowly and thus its $\mathrm{Nu}$ is smaller than P2. Subsequently in drafting stage it decreases again and at the kissing instant it reaches its minimum. During tumbling stage it increases because the relative position between the two cold particles alters from vertical arrangement to inclined arrangement. After tumbling process $\mathbf{P 1}$ is not influenced by the wake of $\mathbf{P 2}$, and thus the two cold particles both obtain a constant $\mathrm{Nu}$ value. For $\alpha=1.0$, the two $\mathrm{Nu}$ curves are almost same before $\mathrm{t}=2000$. In drafting and kissing stage the P1's Nu decreases and P2's Nu increases. In separating stage and hereafter both two Nu values obtain a steady state.

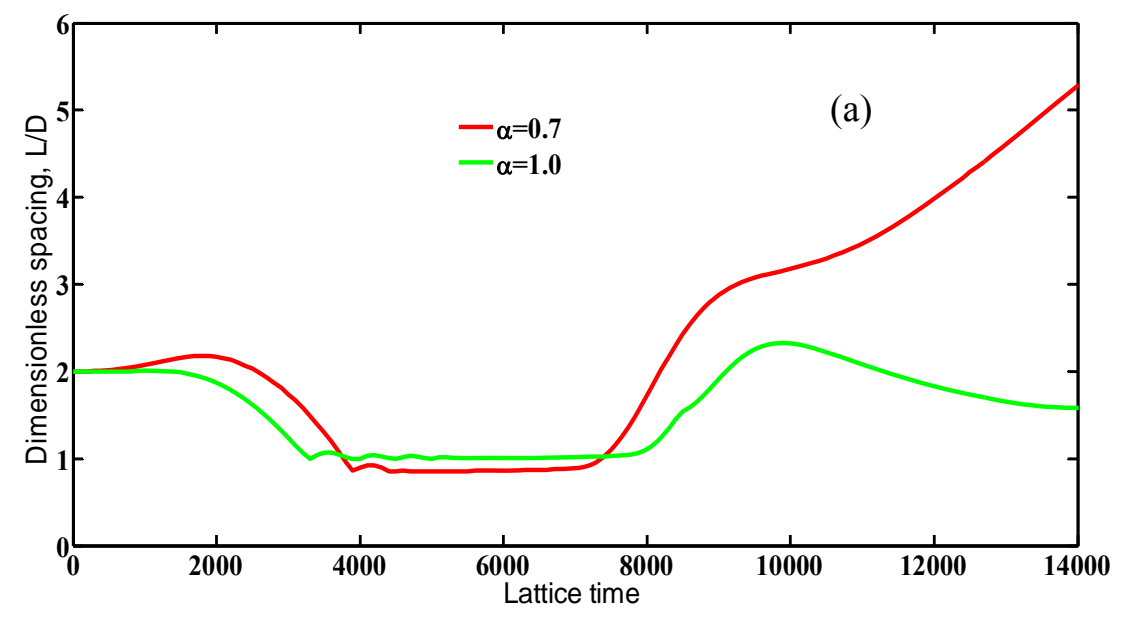



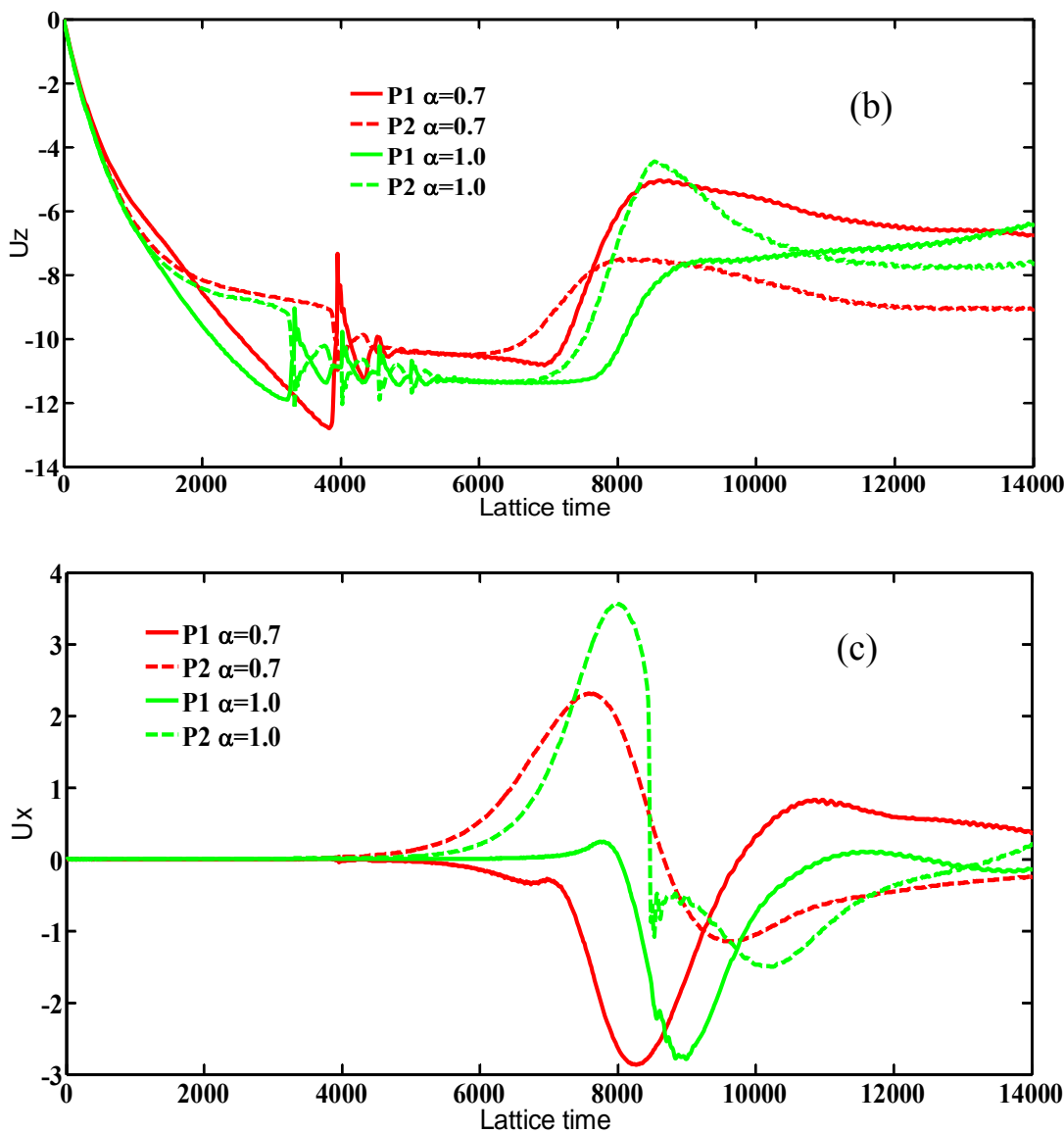

Fig. 5 Variations of (a) dimensionless distance, (b) Z- and (c) X-direction velocities of two cold particles at $\alpha=0.7$ and at $\alpha=1.0$.

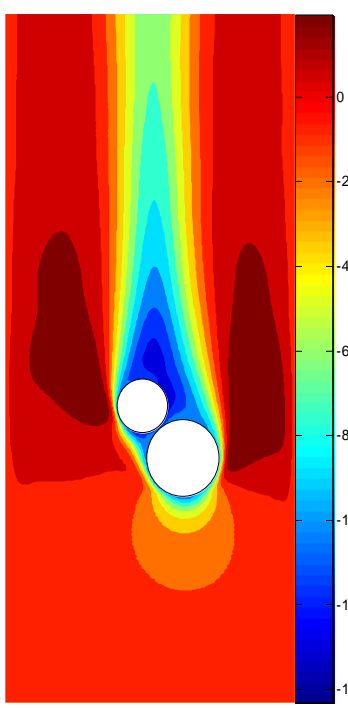

(a)

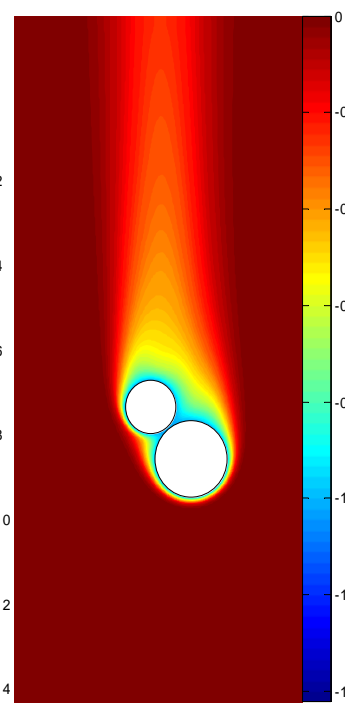

(b)

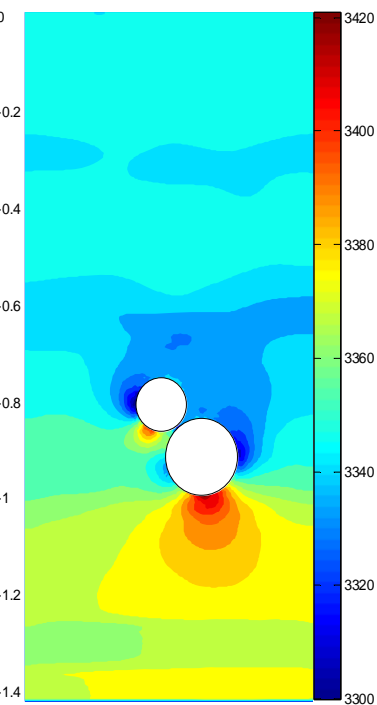

(c)

Fig. 6 Distributions of (a) Z-direction velocity, (b) temperature and (c) pressure contours 
at $\alpha=0.7$ at lattice time $\mathrm{t}=7000$. Only contours on central Y plane are plotted because of symmetry.
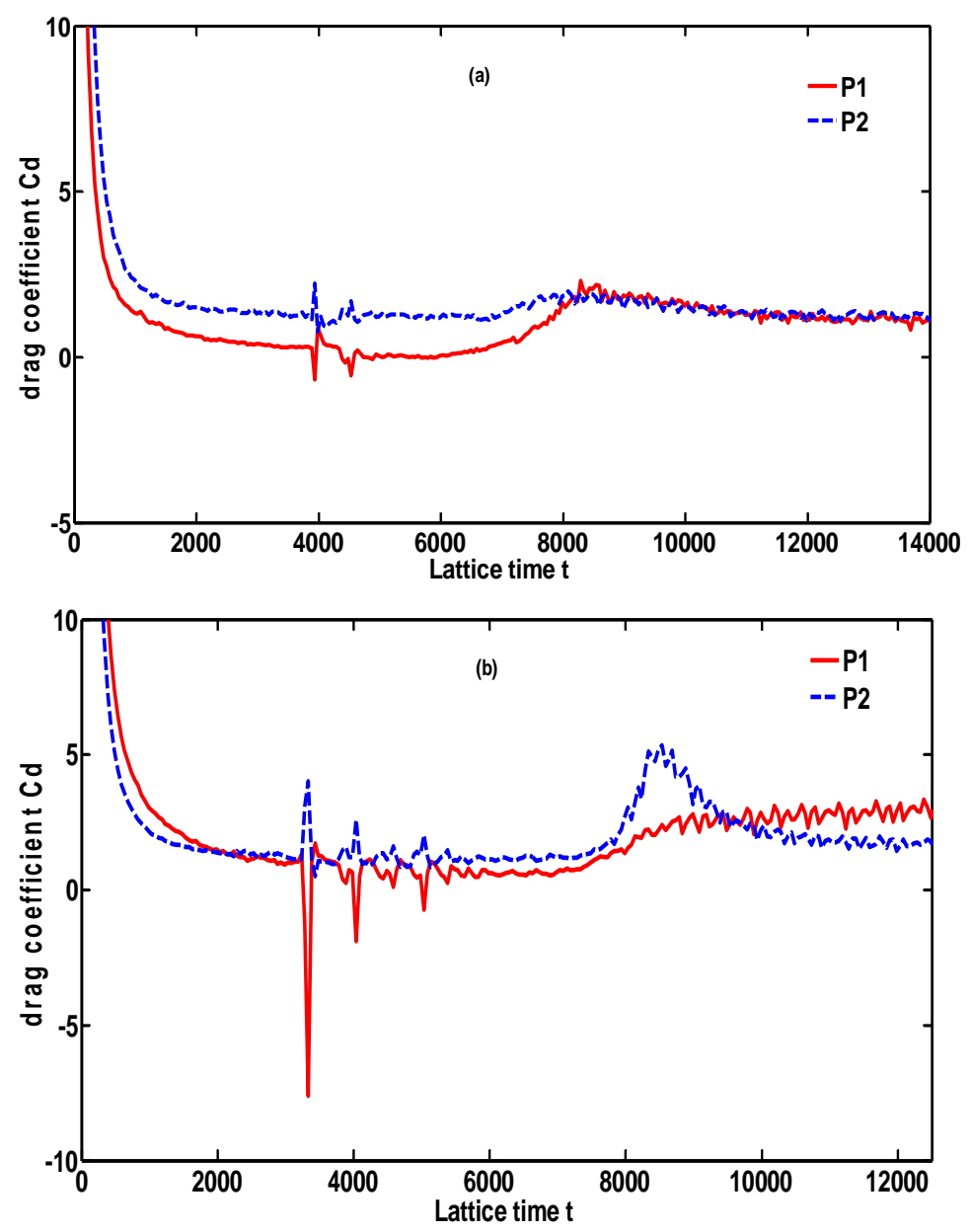

Fig. 7 Variations of drag coefficient $C d$ of two cold particles with time at (a) $\alpha=0.7$ and at (b) $\alpha=1.0$.

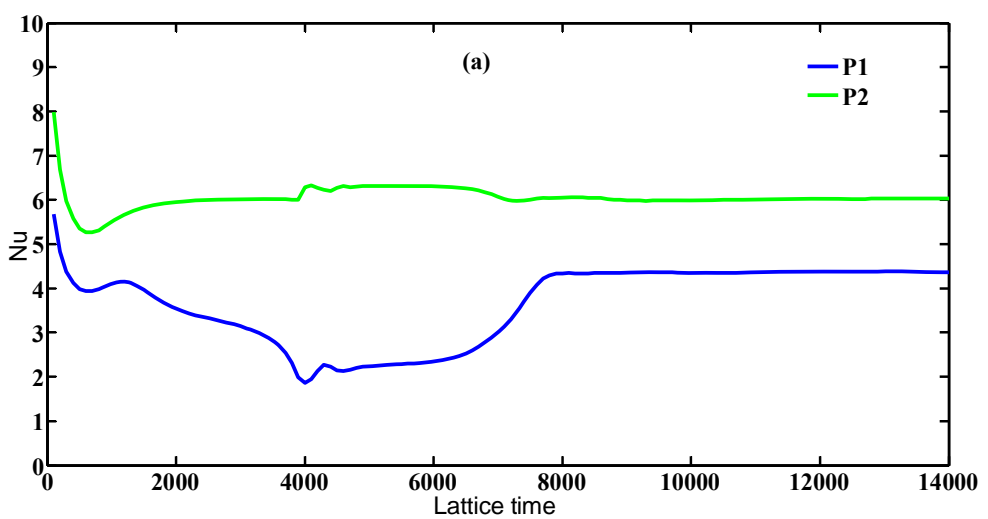




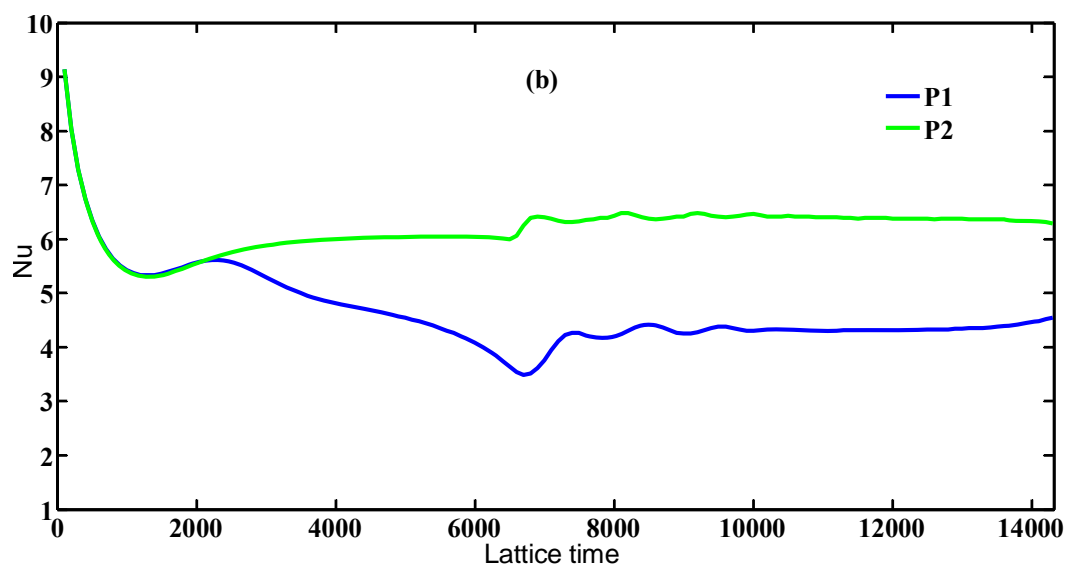

Fig. 8 Variations of Nusselt number $N u$ of two cold particles with time at (a) $\alpha=0.7$ and at (b) $\alpha=1.0$.

\subsection{Sedimentation of two hot particles with different sizes}

In this subsection, size effects on the sedimentation of two hot particles are investigated. Two cases with $\alpha=0.7$ and $\alpha=1.0$ are chosen to illustrate the particle behaviors. Fig. 9 depicts comparisons of distance and velocities of two hot particles in the two cases. With the increasing of size ratio, kissing process occurs earlier and tumbling duration is prolonged. As shown in Fig. 9 (b), for $\alpha=1.0$ P1 settles faster and experiences stronger lubrication force during tumbling than its $\alpha=0.7$ counterpart. After separating stage, for $\alpha=0.7 \mathbf{P 2}$ always settles fast than $\mathbf{P 1}$ and thus their distance is always increased. However, for $\alpha=1.0$, each particle accelerates and decelerates alternately and consequently their distance also increases and decreases alternately (see Fig. 9(a)). During the late period of tumbling, the settling velocities of two hot particles maintain identical but their X-direction velocities increase sharply. This phenomenon may stem from the fact that tumbling alters relative position of two hot particles from initial vertical arrangement to parallel arrangement. 

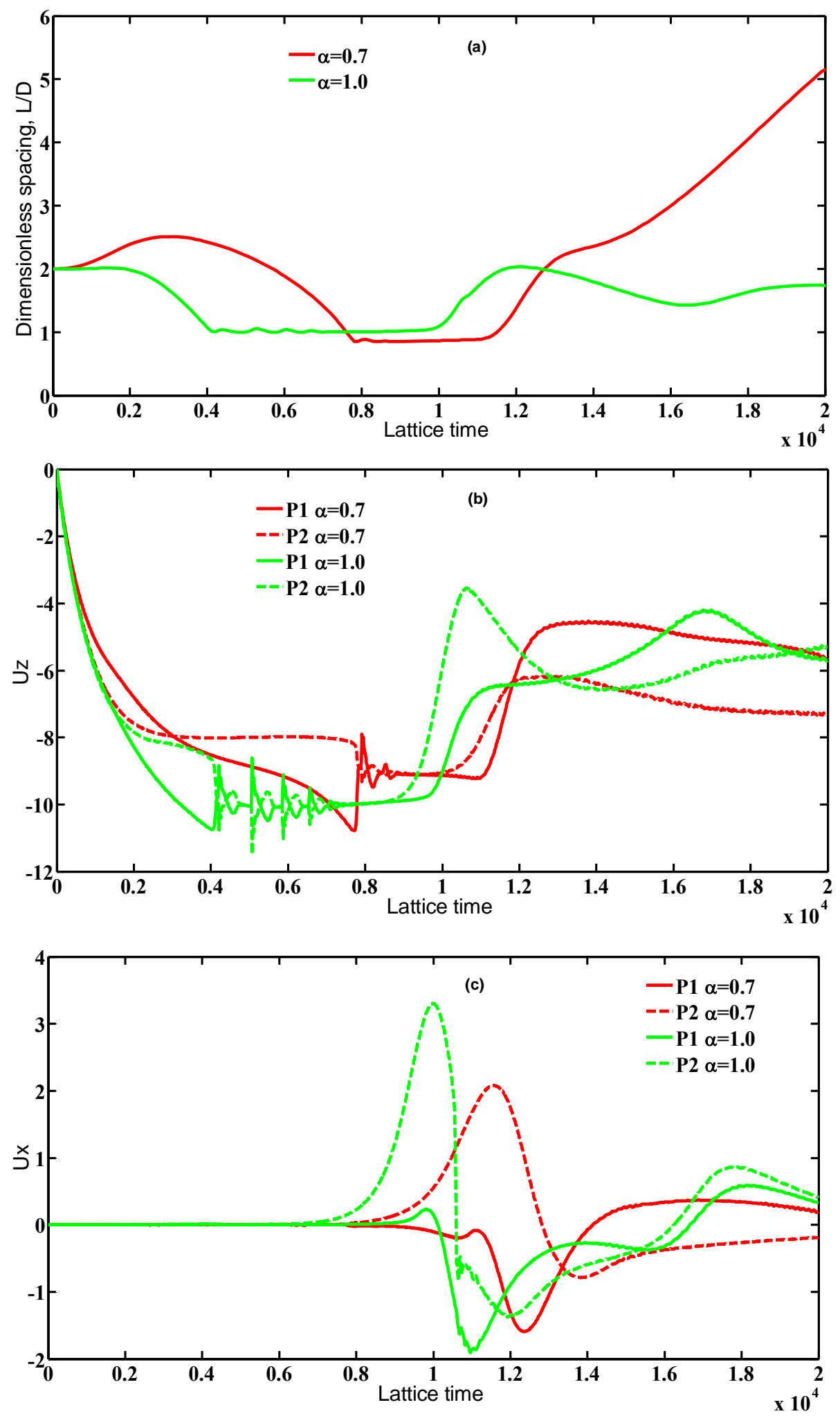

Fig. 9 Comparisons of (a) dimentionless distance, (b) Z-direction and (c) X-direction velocities of two hot particles between at $\alpha=0.7$ and at $\alpha=1.0$.

\subsection{Heat transfer and size effects on the sedimentation of two non-isothermal particles}

In this subsection, the effects of heat transfer on interaction of two non-identical 
particles settling in channel are investigated. Case 1, Case 2 and Case 3 represent two isothermal particles, two hot particles and two cold particles settling in channel, respectively. As pointed out by Ref. [12], for the sedimentation of two isothermal particles with initial vertical position arrangement, there is no DKT process beyond a threshold size ratio. Here we study the effects of heat transfer on this threshold size ratio for the first time. Table 1 lists threshold size ratios $\alpha_{c}$ for the three cases. As expect, the relationship among the three threshold size ratios is $\alpha_{c 3}<\alpha_{c 1}<\alpha_{c 2}$ and their values increase orderly by about 0.1 .

Table 1 Heat transfer effects on threshold size ratio $\alpha_{c}$.

\begin{tabular}{|c|c|}
\hline Cases & Threshold size ratios $\alpha_{c}$ \\
\hline Case 1 & $0.5 \sim 0.6$ \\
\hline Case 2 & $0.6 \sim 0.7$ \\
\hline Case 3 & $0.4 \sim 0.5$ \\
\hline
\end{tabular}

Next we investigate heat transfer effects on the three cases with the same size ratio $\alpha$. Size ratios $\alpha=0.7,0.8,1.0$ are chosen for this investigation. Fig. 10 depicts comparisons of DKT processes among the three cases at various size ratios. Generally speaking, with particle size ratio $\alpha$ increasing, heat transfer effects on the DKT process will be weakened, especially for the case of two hot particles. As depicted in Fig. 10(a), for Case 2 the upper particle slows due to buoyancy force and thus distance between two hot particles increases excessively during initial period. Consequently the upper particle spends more time to catch up with the lower one in drafting stage. However, the lasting time of tumbling duration of the three cases are nearly same. During separating stage, repulsion process between two hot particles is weaker than two other cases. Illustrated by Fig. 10(b) and (c), after separating stage a distance decreasing process appears but no repeated DKT phenomenon happens. It is noted that the variations of DKT curve for Case 2 deviates substantially from other two cases. This indicates heat transfer produces strong effects on the interaction of two hot particles with low size ratios. Fig. 11 plots some instantaneous positions of two settling particles for the three cases. From Fig. 11(b) and (c), one can find that 
wall repulsion effect pushes the two particles to approach each other in horizontal direction. It is the reason why their distances are reduced for a period after separating stage (see Fig. 10(b) and (c)). As shown in Fig. 10(a) and Fig. 11(a), the velocity difference between two particles in settling direction leads to their distance increasing in separating stage. For the situation with size ratio $\alpha=1.0$ shown in Fig. 11(c), after separating stage their trajectories seem like "S" curves. It is also observed in Fig. 11 that against particle size ratio increasing heat transfer effects on the interaction between two non-isothermal particles become weak.
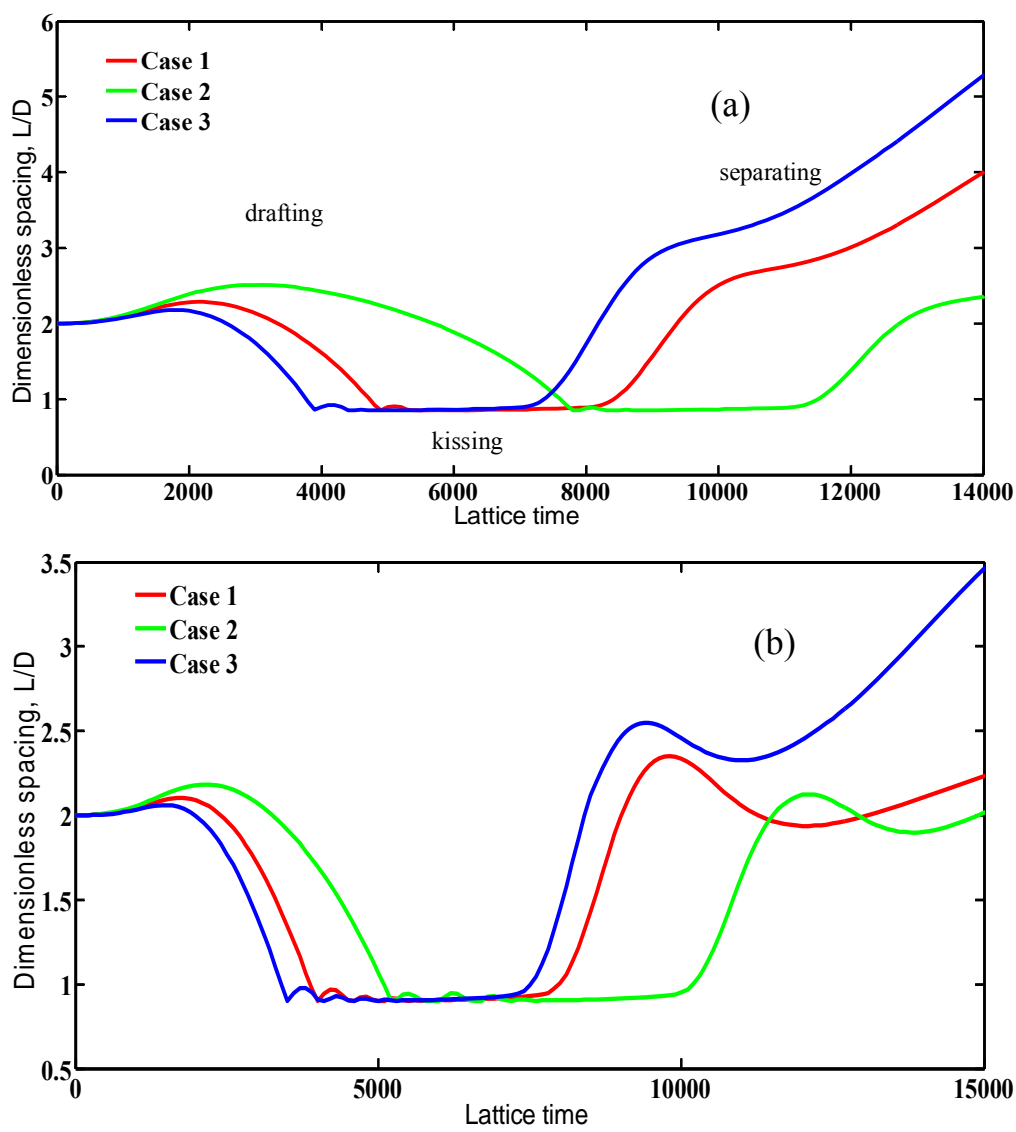


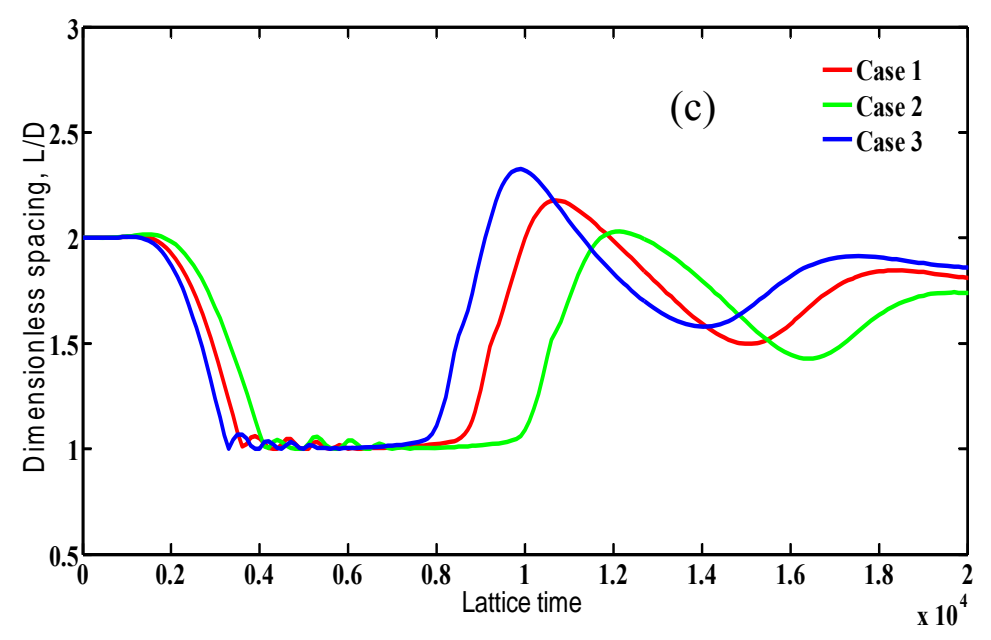

Fig. 10 Comparisons of dimensionless spacing of two particles among the three cases.

(a) $\alpha=0.7$, (b) $\alpha=0.8$ and (c) $\alpha=1.0$.
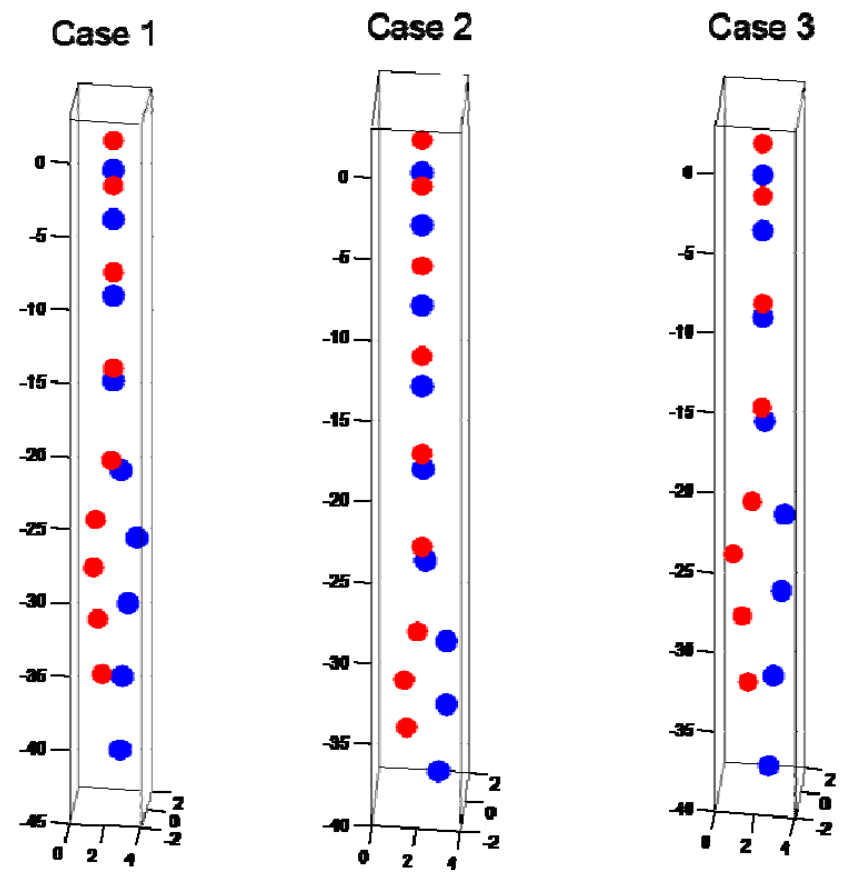

(a) 

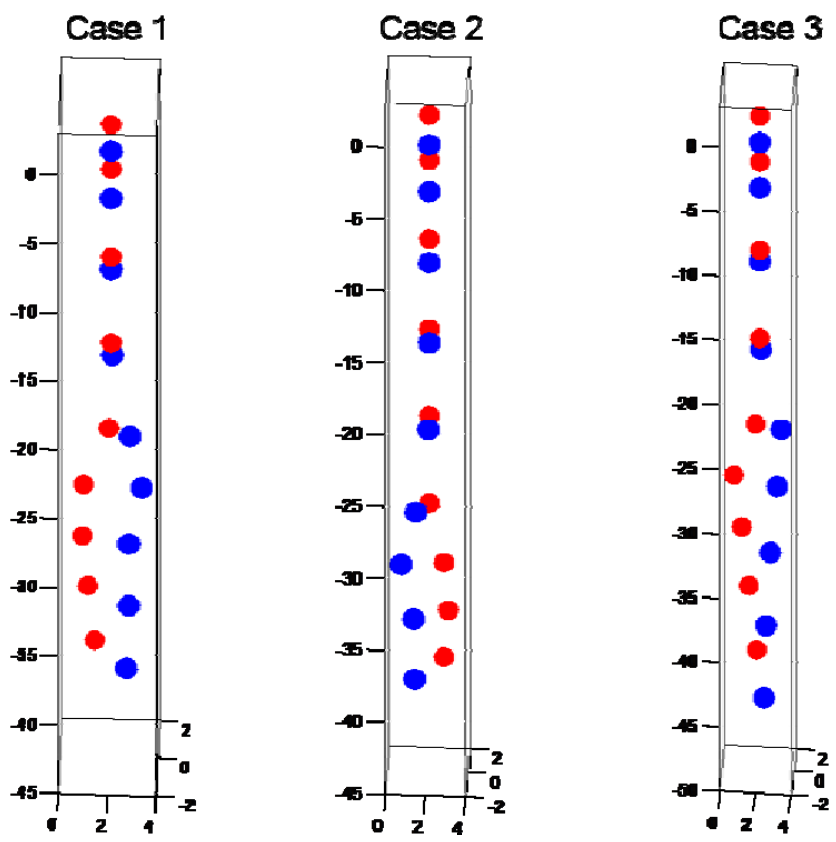

(b)

\section{Case 1}

Case 2

Case 3
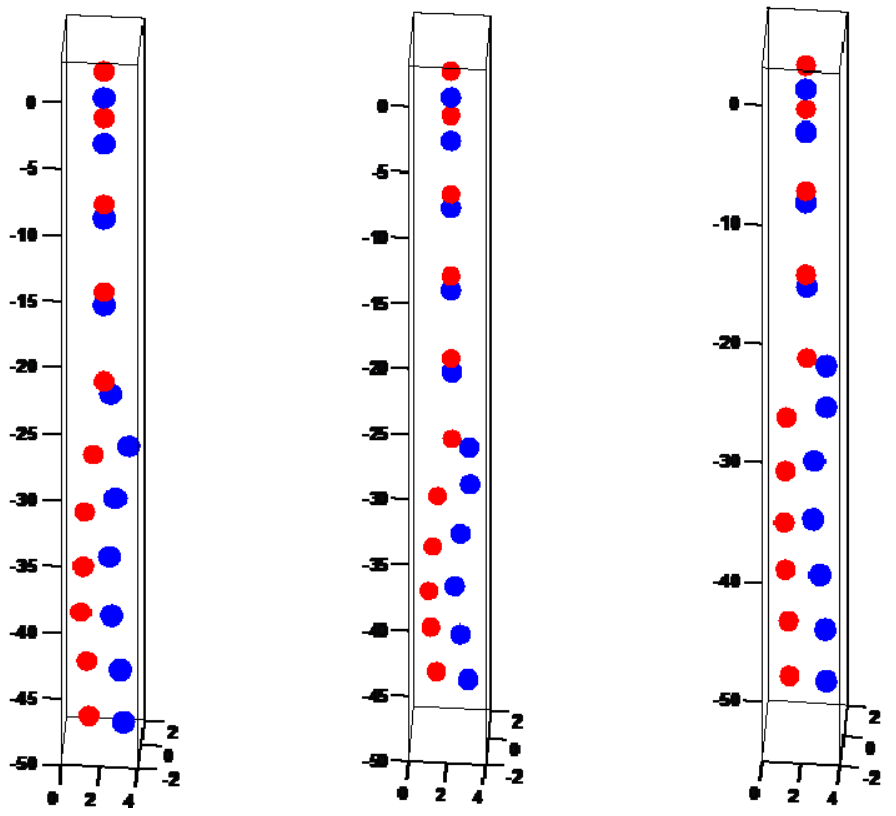

(c)

Fig. 11 Trajectories of two particles settling in vertical channel for the three cases. (a) $\alpha=0.7$, (b) $\alpha=0.8$ and (c) $\alpha=1.0$.

\section{Conclusion}

The size ratio effects on the sedimentation of two spherical particles with heat transfer are investigated using LB-IB method for the first time. In this work three 
cases are investigated: (1) sedimentation of two isothermal particles, (2) sedimentation of two hot particles and (3) sedimentation of two cold particles. Through our analysis, we can draw the following conclusions:

1) The size ratio effects on the DKT between two non-isothermal particles are significant. For two hot particles and two cold particles, tumbling duration is prolonged with the size ratio increasing, which is consistent with their isothermal counterpart. For all three cases, there is little difference of drag coefficient and Nusselt number between two non-identical particles when particle behaviors reach steady state.

2) The differences of threshold size ratio among the above three cases are significant. When the small particle is placed above the large one initially, the threshold size ratios for two cold particles, two isothermal particles and two hot particles are increased orderly.

3) Heat transfer produces a strong effect on the sedimentation of two hot particles with low particle size ratios. Moreover against particle size ratio increasing heat transfer effects on the interaction between two non-isothermal particles become weak.

\section{Acknowledgments}

This work has received funding from the Universidad Carlos III de Madrid, the European Union's Seventh Framework Programme for research, technological development and demonstration under grant agreement No. 600371, el Ministerio de Economía y Competitividad (COFUND2014-51509), el Ministerio de Educación, cultura y Deporte (CEI-15-17) and Banco Santander. We also acknowledge the support from the British Newton Alumni Fellowship Scheme, the National Natural Science Foundation of China (Grant No. 51176061). 


\section{References}

[1]A.F. Fortes, D.D. Joseph, T.S. Lundgren, Nonlinear mechanics of fluidization of beds of spherical particles, J. Fluid Mech. 177 (1987) 467-483.

[2]J. Feng, H H Hu, D.D. Joseph, Direct simulation of initial value problems for the motion of solid bodies in a Newtonian fluid Part 1. Sedimentation, J. Fluid Mech. 261 (1994) 95-134.

[3]Gan H, Chang J, Feng J J, Direct numerical simulation of the sedimentation of solid particles with thermal convection, Journal of Fluid Mechanics 481 (2003) 385-411.

[4]Zahra Hashemi, Omid Abouali, Reza Kamali, Three dimensional thermal Lattice Boltzmann simulation of heating/cooling spheres falling in a Newtonian liquid, International Journal of Thermal Sciences 82 (2014) 23-33.

[5]Henrik Ström, Srdjan Sasic, Detailed simulations of the effect of particle deformation and particle-fluid heat transfer on particle-particle interactions in liquids, Procedia Engineering 102 (2015) 1563-1572.

[6] Chuansheng Cao, Sheng Chen, Jing Li, Zhaohui Liu, Lu Zha, Sheng Bao, Chuguang Zheng, Simulating the interactions of two freely settling spherical particles in Newtonian fluid using lattice-Boltzmann method, Applied Mathematics and Computation 250 (2015) 533-551.

[7]Bo Yang, Sheng Chen, Chuansheng Cao, Zhaohui Liu, Chuguang Zheng, Lattice Boltzmann simulation of two cold particles settling in Newtonian fluid with thermal convection, International Journal of Heat and Mass Transfer 93 (2016) 477-490.

[8]Johnson A, Tezduyar TE, 3D simulation of fluid-particle interactions with the number of particles reaching 100, Comput Methods Appl Mech Eng 145(1997) 301-321.

[9]Mukundakrishnan K, Hu HH, Ayyaswamy PS, The dynamics of two spherical particles in a confined rotating flow: pedaling motion, J Fluid Mech 599 (2008) 169-204.

[10] Shao XM, Liu Y, Yu ZS, Interactions between two sedimenting particles with different sizes, Appl Math Mech-Engl 26 (2005) 407-414.

[11] Wang L, Guo ZL, Mi JC, Drafting, kissing and tumbling process of two particles with different sizes, Comput Fluids, 96 (2014) 20-34.

[12] Chuan-Chieh Liao, Wen-Wei Hsiao, Ting-Yu Lin, Chao-An Lin, Simulations of two sedimenting-interacting spheres with different sizes and initial configurations using immersed boundary method, Comput Mech 55 (2015) 1191-1200.

[13] A.J.C. Ladd, Sedimentation of homogeneous suspensions of non-Brownian spheres, Phys. Fluids 9 (1997) 491-499.

[14] C.K. Aidun, L. Yannan, E.J. Ding, Direct analysis of particulate suspensions with inertia using the discrete Boltzmann equation, J. Fluid Mech. 373 (1998) 287-311.

[15] O. Behrend, Solide uid boundaries in particle suspension simulations via the lattice Boltzmann method, Phys. Rev. E 52 (1995) 1164.

[16] D. Qi, Lattice-Boltzmann simulations of particles in nonzero-Reynolds number flows, J. Fluid Mech. 385 (1999) 41. 
[17] Guo ZL, Zheng CG, Shi BC, Discrete lattice effects on the forcing term in the lattice Boltzmann method, Phys Rev E 65 (4) (2002):046308.

[18] Shin K. Kang, Yassin A. Hassan, A comparative study of direct-forcing immersed boundary-lattice Boltzmann methods for stationary complex boundaries, Int. J. Numer. Meth. Fluids 66 (2011) 1132-1158.

[19] Kang S K, Hassan Y A, A direct-forcing immersed boundary method for the thermal lattice Boltzmann method, Computers \& Fluids 49 (2011) 36-45.

[20] Jungwoo Kim, Dongjoo Kim, Haecheon Choi, An Immersed-Boundary Finite-Volume Method for Simulations of Flow in Complex Geometries, Journal of Computational Physics, 171 (2001) 132-150.

[21] J. Kromkamp, D. v. den Ende, D. Kandhai, R. van der Sman, R. Boom, Lattice Boltzmann simulation of 2D and 3D non-Brownian suspensions in Couette flow, Chemical Engineering Science, 61 (2006) $858-873$.

[22] Nguyen, N.-Q., Ladd, A.J.C., Lubrication corrections for Lattice-Boltzmann simulations of particle suspensions, Physical Review E 66 (2002):046708.

[23] C. Dan, A. Wachs, Direct numerical simulation of particulate flow with heat transfer, Int. J. Heat Fluid Flow, 31 (2010) 1050-1057.

[24] Yu Z, Phan-Thien N, Fan Y, Tanner RI, Viscoelastic mobility problem of a system of particles, J Non Newtonian Fluid Mech 104(2002) 87-124. 